\title{
Vital Exhaustion and Incidence of Dementia: Results from the Copenhagen City Heart Study
}

\author{
Sabrina Islamoska ${ }^{\mathrm{a}, *}$, Kazi Ishtiak-Ahmed ${ }^{\mathrm{a}}$, Åse Marie Hansen ${ }^{\mathrm{a}, \mathrm{b}}$, Matias Brødsgaard Grynderup ${ }^{\mathrm{a}}$, \\ Erik Lykke Mortensen ${ }^{\mathrm{a}, \mathrm{c}}$, Anne Helene Garde ${ }^{\mathrm{a}, \mathrm{b}}$, Finn Gyntelberg ${ }^{\mathrm{b}}$, Eva Irene Bossano Prescott ${ }^{\mathrm{d}}$, \\ Eszter Török ${ }^{\mathrm{a}}$, Gunhild Waldemar ${ }^{\mathrm{e}}$ and Kirsten Nabe-Nielsen ${ }^{\mathrm{a}}$ \\ ${ }^{a}$ Department of Public Health, University of Copenhagen, Copenhagen, Denmark \\ ${ }^{\mathrm{b}}$ The National Research Centre for the Working Environment, Copenhagen, Denmark \\ ${ }^{\mathrm{c} C e n t e r ~ f o r ~ H e a l t h y ~ A g i n g, ~ U n i v e r s i t y ~ o f ~ C o p e n h a g e n, ~ C o p e n h a g e n, ~ D e n m a r k ~}$ \\ ${ }^{\mathrm{d}}$ Department of Cardiology, Bispebjerg Hospital, Copenhagen, Denmark \\ e Danish Dementia Research Centre, Rigshospitalet, University of Copenhagen, Copenhagen, Denmark
}

Handling Associate Editor: Shireen Sindi

Accepted 5 November 2018

\begin{abstract}
.
Background: Psychological distress is potentially linked to the risk of dementia through neurologic and cardiovascular mechanisms. Vital exhaustion (VE) is a mental state of psychological distress, which could be a risk factor for dementia.

Objective: To investigate whether VE is a risk factor for dementia in later life.

Methods: We used data from 6,807 participants attending the third survey of the Copenhagen City Heart Study in 1991-1994. VE was assessed by 17 symptoms (score: 0-17) from the Maastricht Questionnaire. Information on dementia was obtained from national registers. Risk time for dementia was counted from five years after VE assessment for participants $>55$ years at the time of VE assessment. For younger participants, risk time for dementia was counted from the year they turned 60 years and onwards. Participants were followed until 2016. We used Poisson regression to calculate incidence rate ratios (IRR) and their $95 \%$ confidence intervals $(\mathrm{CI})$.

Results: During an average follow-up of 10 years, 872 participants were registered with dementia. We found a doseresponse relation between the number of VE symptoms and the incidence of dementia. For every additional VE symptom, the dementia incidence increased by $2 \%$ (IRR $=1.024 ; 95 \%$ CI: 1.004-1.043). Adjustment for socio-demographic and healthrelated factors did not change the results substantially. Neither did stratification by age, sex, educational level, and marital status.

Conclusion: We found evidence that VE is a risk factor for dementia. Our sensitivity analyses supported that this association was not only due to VE being a potential prodromal sign of dementia.
\end{abstract}

Keywords: Dementia, mental health, psychological stress, subjective health complaint

\footnotetext{
*Correspondence to: Sabrina Islamoska, Department of Public Health, University of Copenhagen, Øster Farimagsgade 5,
}

1014 Copenhagen, Denmark. Tel.: +45 3533 6070; E-mail: saim@sund.ku.dk. 


\section{INTRODUCTION}

Dementia is a common disorder affecting memory, behavior, emotions, and thinking [1, 2]. Dementia also interferes with independence in everyday activities [1, 2]. In 2015, 46.8 million individuals were living with dementia worldwide, and the number of affected individuals is estimated to double every 20 years from 2015 and onwards [3]. Dementia is strongly age-related, and at age 60-64 years, the incidence of dementia is estimated to be 3.9 per 1,000 person-years, while it is 104.8 per 1,000 person-years at age $90+$ years [3].

Previous studies have suggested that psychological distress, operationalized, e.g., as feelings of anxiety, depression, irritability, and reactions to external stressors in one's everyday life, is associated with an increased risk of dementia [4-10]. Psychological distress can be defined as a state of emotional suffering sometimes accompanied by somatic symptoms [11]. Experiencing psychological distress in specific periods during life may affect cognitive health [12]. When the physiological stress response is triggered, hypothalamic-pituitary-adrenal axis activity fosters an excessive production of cortisol, which potentially leads to hippocampal damage [4, 5, 9, 12-15]. Thereby, the stress response can affect cognitive function and the risk of dementia.

Vital exhaustion (VE) is a mental state of psychological distress and is proposed by Appels and Mulder to denote premonitory symptoms of myocardial infarction [16, 17]. VE is suggested to be a potential response to unsolvable problems in individuals' lives, in particular when being incapable of adapting to prolonged exposure to psychological stressors [17-19]. Previous studies have reported that VE is associated with cardiovascular disease (CVD), metabolic syndrome, elevated platelet count, all-cause mortality, chronic obstructive pulmonary disease, weight gain, and risk of obesity [19-24]. VE covers both somatic and cognitive complaints of unusual fatigue, increased irritability and feelings of demoralization [16, 17, 19]. VE shares similar symptoms with depression, which is a risk factor for dementia [2, 25]. However, VE has been proposed to represent a more somatic dimension of distress and the cognitive/affective symptoms of depression have shown to be less apparent in VE [20,26]. Based on previous studies on psychological distress and dementia, more longitudinal studies are needed to contribute to a broader understanding of the role of psychological distress in relation to later cognitive health $[6,7,27]$. Therefore, in the present study, we investigate whether VE is a risk factor for dementia in later life.

\section{MATERIALS AND METHODS}

\section{Study population and design}

The Copenhagen City Heart Study (CCHS) is a large population-based study established in 1975 [28]. A sample of 20,000 women and men was randomly drawn by using personal identification numbers from 90,000 inhabitants aged 20 years and above living in the Copenhagen area in Denmark [28]. The inhabitants were invited to participate in four examinations at different time points in the years 1976-1978, 1981-1983, 1991-1994, and 2001-2003 [28]. For the present paper, data on all participants born in 1956 or earlier were available. The primary aim of CCHS was the prevention of coronary heart disease and stroke; however, many aspects of other conditions were added during the study's follow-up surveys [28]. CCHS is described in detail elsewhere [28]. Our study population was based on participants enrolled at baseline in the third survey of CCHS in 1991-1994 $(N=10,135)$ in which participants' VE symptoms were registered. The response rate of the third survey was $61.2 \%$ [28] and participants included in this study were followed until the end of 2016 with a very low loss to follow-up due to emigration $(n=3)$. In order to be at risk of dementia, we initially restricted our study population to 8,570 participants who were born in 1956 or earlier and who would therefore turn 60 years before end of follow-up in 2016. We made this restriction in order to include participants who reached an age during follow-up where they would be at risk of dementia. Furthermore, it has been shown that the validity of dementia diagnoses in younger patients is poor [29, 30]. We also excluded participants who were 80 years or older when they responded to questions about VE, because of the high risk of undiagnosed dementia in this age group with reverse causation as a consequence. VE was assessed in 1991-1994 (referred to as baseline). To reduce reverse causation, we postponed start of follow-up with five years from the time of baseline participation (i.e., 1995-1999). This means that participants who were diagnosed with dementia within the first five years after baseline were excluded. Furthermore, for those who were younger than 60 years in 1995-1999, start of follow-up was 


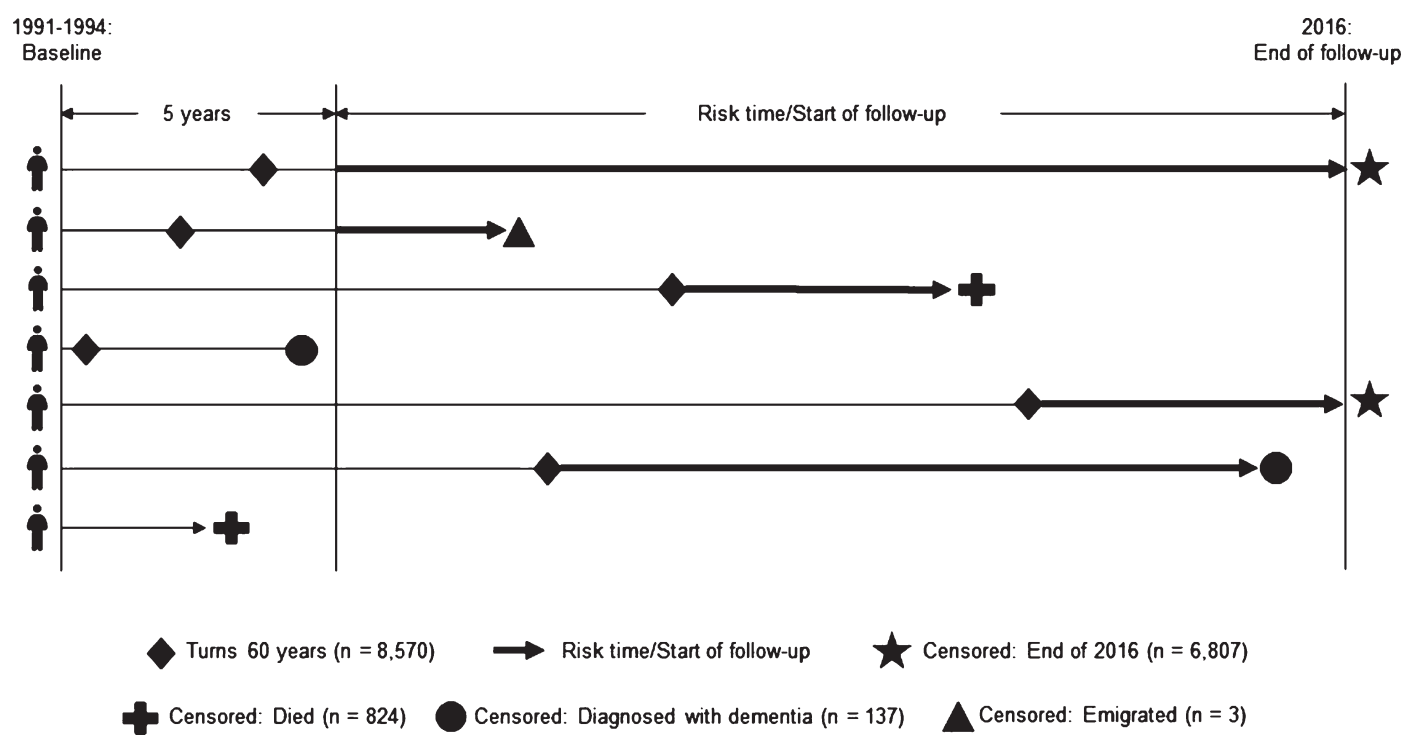

Fig. 1. Study design.

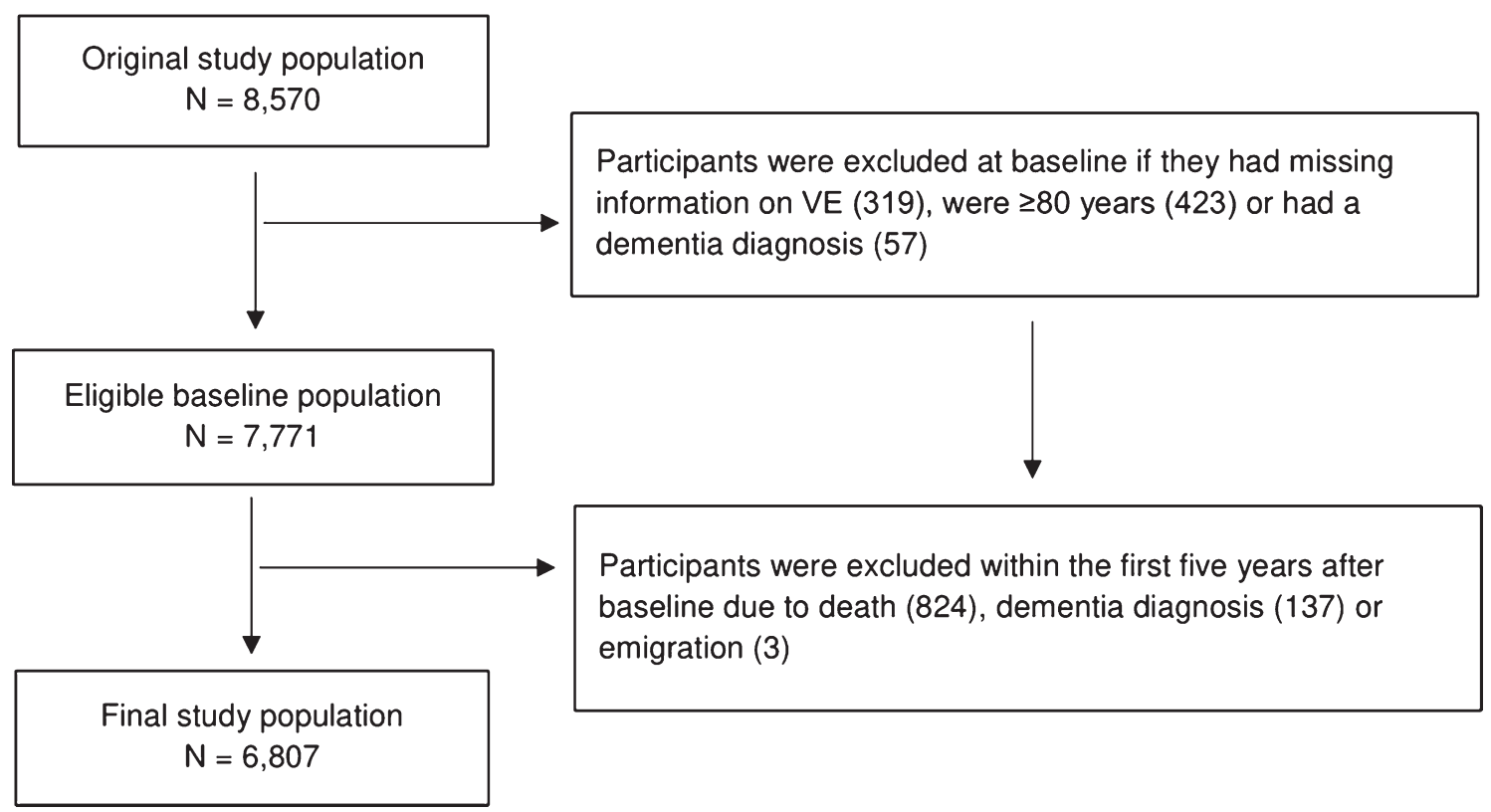

Fig. 2. Flow chart of the final study population selected for analyses $(\mathrm{N}=6,807)$.

further postponed until they turned 60 years. This latter step was taken in order to only include risk time for participants who were at risk of dementia. A final requirement was that participants needed to be free of dementia (i.e., not registered with a dementia diagnosis) when starting to contribute with risk time to our analyses. Participants were followed until death, emigration, dementia diagnosis, or end of followup in 2016, whichever came first (Fig. 1). Thus, our final study population consisted of 6,807 participants (Fig. 2). Through an encrypted personal identification number, the data from CCHS were linked to information from Danish national registers.

\section{Vital exhaustion}

VE was assessed by a 17-item questionnaire based on the original Maastricht Questionnaire (MQ) [16, 
Table 1

The 17 vital exhaustion symptoms from the Maastricht Questionnaire

Do you ...

Often feel tired?

Feel altogether weak?

Feel you have not accomplished much recently?

Sometimes have difficulty coping?

Think you have come to a dead end?

Lately feel listless?

Have feelings of hopelessness recently?

Lately have difficulties concentrating?

Do little things irritate you more than they used to?

Feel that you want to give up?

Feel fine?

Sometimes feel your body is like a battery running out?

Sometimes wish you were dead?

At the moment feel that you do not have what it takes?

Feel dejected?

Sometimes just feel like crying?

Ever wake up with a feeling of exhaustion?

17, 31] (Table 1). Participants were presented with the $17 \mathrm{VE}$ symptoms and were asked how they had been feeling within the past six months. The response options of VE were scored 1 for 'Yes' and 0 for ' $N o$ ' and 'I do not know' except for the item 'Feel fine' which was reverse scored [17,31]. In accordance with previously applied categorization, we divided VE into four categories based on the total sum of symptoms: 0 (reference), 1-4 (low), 5-9 (medium), and 10-17 (high) [31]. The main analyses were conducted for the total VE score (range: 0-17) both as a continuous variable and a categorical variable with the four categories, respectively.

\section{Dementia}

Dementia was defined from one of the following whichever came first: 1) First registration of a dementia diagnosis in the Danish National Patient Register [32], the Danish Psychiatric Central Research Register [33], and the Danish Register of Causes of Death [34] using the International Classification of Diseases (ICD) codes from the 8th (ICD-8) and 10th (ICD-10) revision: unspecified dementia (ICD-8: 290.09-19; ICD-10: F03.9, G31.9), Alzheimer's disease (AD) (ICD-8: 290.10; ICD-10: F00.0-00.9, G30.0-30.9), vascular dementia (ICD-8: 293.09-19; ICD-10: F01.0-01.9), frontotemporal dementia (ICD-8: 290.11; ICD-10: F02.0), and Lewy body dementia (ICD-10: G31.8). The ICD-8 system was in use until 1993, and the ICD10 system was introduced in 1994. This means that we used ICD-8 codes to identify and exclude participants with dementia at the time of the baseline assessment of VE in 1991-1994; 2) First prescription and purchase of an anti-dementia drug such as cholinesterase-inhibitors (donepezil or, galantamine, or rivastigmine) or glutamate-receptor antagonists (memantine), which are used in the treatment of $\mathrm{AD}$, Lewy body dementia, and dementia in Parkinson's disease. Based on these prescription data from the Danish National Prescription Registry [35], we identified patients treated with anti-dementia medication, but who are not registered with a dementia diagnosis. In order to receive a prescription for subsidized dementia medication in Denmark, patients have to be diagnosed with dementia by a specialist in geriatrics, neurology or psychiatry.

\section{Covariates}

In our statistical analyses, we chose to adjust for covariates that have been shown to be associated with dementia (references in the following paragraphs support the association between the chosen covariates and dementia). Baseline data were obtained from CCHS in 1991-1994 and follow-up data from registers were obtained until 2016.

\section{Socio-demographic factors}

From the national registers, we obtained baseline information on age and sex [2]. From CCHS data in 1991-1994, we obtained information on marital status [36] categorized as being married (married/cohabiting)/unmarried (not married; divorced/separated; widow/widower), and the highest attained educational level [2] defined as low educational level (primary school), medium educational level (upper secondary education; business high school; vocational education and training), and high educational level (short-term further education; middle-range education; bachelor's degree; extended education; and research degree). From the national registers, we also included information on educational level for participants who had missing information in CCHS.

\section{Lifestyle factors}

From CCHS, we obtained baseline information on tobacco smoking [2] categorized as current smoker/past smoker/non-smoker; alcohol intake [2] measured as a continuous variable (unit per week); body mass index (BMI) classified according to standard defined cut-off points as underweight $\left(<18.5 \mathrm{~kg} / \mathrm{m}^{2}\right)$, normal $\left(18.5-24.9 \mathrm{~kg} / \mathrm{m}^{2}\right)$, 
overweight (25-29.9 kg/m $\left.{ }^{2}\right)$, and obese $\left(>30 \mathrm{~kg} / \mathrm{m}^{2}\right)$; physical activity [2] in leisure time categorized into four categories as sedentary (light activity less than 2 hours a week), light (light activity $2-4$ hours a week), moderate (light activity for more than 4 hours or vigorous activity 2-4 hours a week), and hard (vigorous activity for more than 4 hours a week or regular hard physical activity); sleep medication [37] defined as daily or almost daily use of sleep medication categorized as yes/no; total cholesterol [2] in $\mathrm{mmol} / \mathrm{L}$; and blood pressure [2] included as systolic and diastolic blood pressure $(\mathrm{mmHg}$ ) (blood samples were measured non-fasting).

\section{Comorbidities}

From the national registers, diagnoses were obtained on diabetes mellitus, CVD (coronary heart diseases and cerebrovascular diseases), and mental disorders defined as a record of psychiatric hospitalization (schizophrenia, schizotypal or schizoaffective disorders, affective psychoses or disorders, paranoid states, other psychoses, unspecified psychoses, manic episodes, nervousness and debility, depressive neuroses, recurrent depressive disorders, delusional disorders and acute and transient psychotic disorders) [2]. We only used information about the different diagnoses for comorbidities occurring before baseline.

\section{Statistical analyses}

In the descriptive statistics, chi-square tests were applied to investigate differences across the four VE categories in the categorical variables, and ANOVA tests were applied to investigate differences in the mean scores of the continuous variables. Follow-up analyses were performed by Poisson regression using person-years (on log-scale) as off-set with VE in four categories and as a continuous scale, respectively. The purpose of using both measures of VE was to investigate the incidence of dementia when considering both one-symptom changes in VE and also potential differences in reporting low, medium, and high levels of VE based on specific numbers of symptoms. The followup time was computed into one-year intervals including three different time scales: age, time since VE assessment (i.e., participation in the survey), and calendar year. The start of follow-up time was counted when at least five years had passed since baseline and the participants had turned at least 60 years.

We adjusted for covariates in four different models. A crude model was applied without adjusting for any covariates (Model 1). In Model 2 (our main model), we adjusted for socio-demographic factors (current age, sex, educational level, marital status), mental disorders, calendar time, and time since VE assessment. In Model 3, we further adjusted for lifestyle factors (tobacco smoking, alcohol intake, BMI, physical activity, sleep medication, total cholesterol, systolic blood pressure). Finally, in Model 4, we also adjusted for previous history of diabetes mellitus and CVD. Since lifestyle factors and history of diabetes mellitus and CVD could act as both confounders and mediators in the association between VE and dementia, we included these factors in separate models (Model 3 and 4).

In addition, we performed analyses stratified by age, sex, educational level, and marital status to investigate potential differences in the association between VE and the incidence of dementia within these groups [2]. For these analyses, we dichotomized the age variable according to the mean age of 60 years at baseline, i.e., $<60$ years and $\geq 60$ years.

We performed sensitivity analyses in which we postponed the start of follow-up until 10, 15 and 20 years after the VE assessment to test whether the association between VE and dementia remained robust to this methodological choice. Using Pearson correlation analysis, we also investigated how stable VE was in participants who reported VE at baseline in 1991-1994 and 10 years later at CCHS's next survey in 2001-2003.

\section{RESULTS}

\section{Characteristics of the study population}

In the overall study population, the mean age at the time of VE assessment was 60 years $(\mathrm{SD}=10.7)$, $58.5 \%$ of the population was women and the mean VE score was $3.0(\mathrm{SD}=3.6)$. Approximately $25 \%$ of the study population reported medium (5-9 symptoms) and high VE (10-17 symptoms) scores (Table 2). Participants with higher VE scores were more often women, unmarried, smokers, sedentary during leisure time, and daily users of sleep medication, and they more often had a low educational level and previous mental disorders or CVD.

\section{Main analyses}

During an average follow-up time of 10 years $(\mathrm{SD}=5.7), 13 \%(n=872)$ of the participants were diagnosed with dementia. The mean age of the 
Table 2

Baseline characteristics of the study population according to their vital exhaustion (VE) score shown in prevalence and means with standard deviations (SD) $(\mathrm{N}=6,807)$

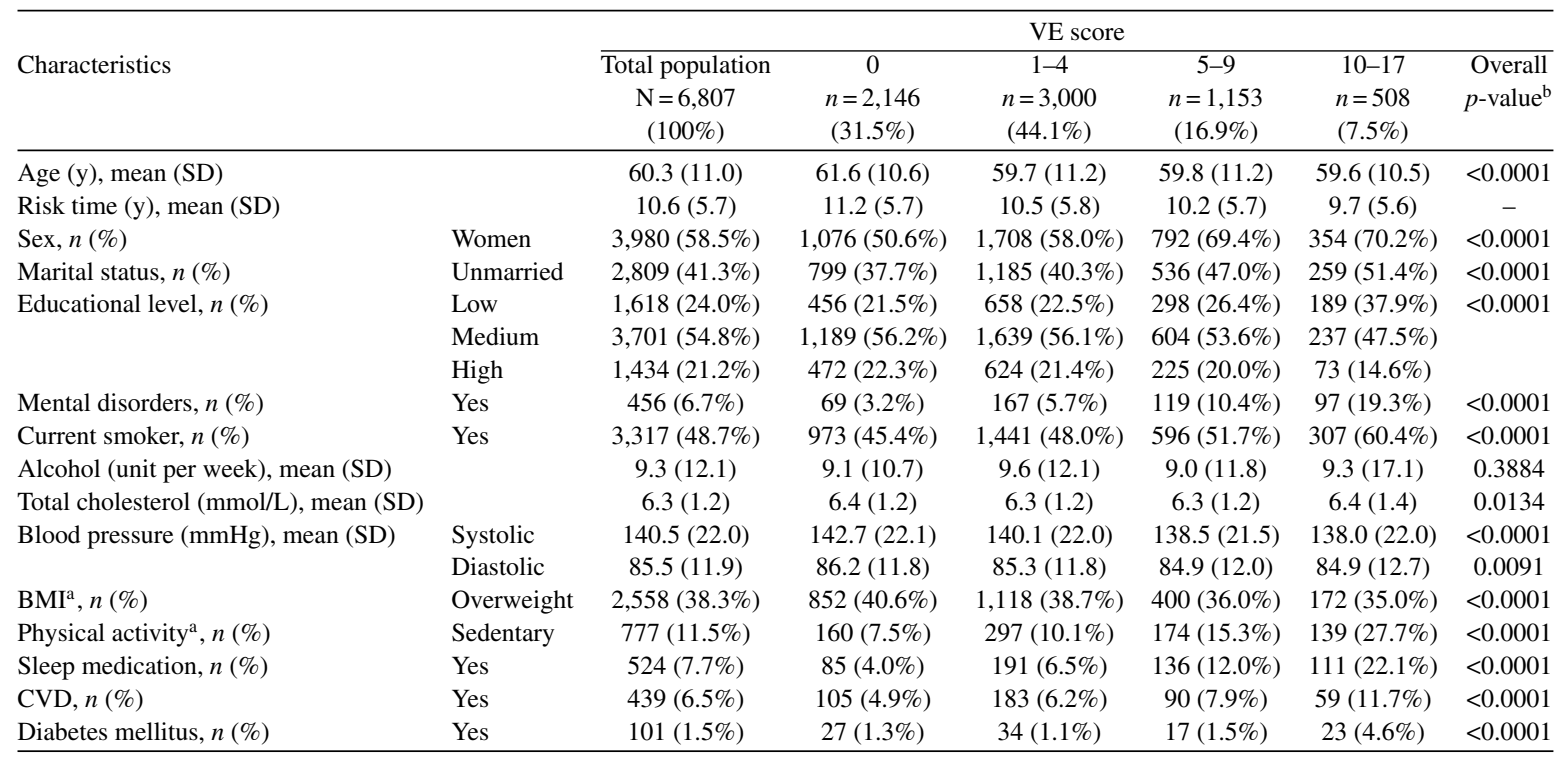

a BMI: Body mass index categorized as underweight $\left(<18.5 \mathrm{~kg} / \mathrm{m}^{2}\right)$, normal $\left(18.5-24.9 \mathrm{~kg} / \mathrm{m}^{2}\right)$, overweight $\left(25-29.9 \mathrm{~kg} / \mathrm{m}^{2}\right)$, and obese $\left(>30 \mathrm{~kg} / \mathrm{m}^{2}\right)$. Physical activity in leisure time categorized as sedentary (light activity less than 2 hours a week), light (light activity $2-4$ hours a week), moderate (light activity for more than 4 hours or vigorous activity 2-4 hours a week), and hard (vigorous activity for more than 4 hours a week or regular hard physical activity). ${ }^{\mathrm{b}} p$-values were based on chi-square tests for categorical variables and ANOVA tests for continuous variables. The $p$-value for the educational variable was calculated based on all three educational levels.

participants when being diagnosed with dementia was 82 years $(\mathrm{SD}=6.9)$.

In Table 3, the results from the analyses with the categorical VE variable showed that there was a dose-response relationship between levels of VE symptoms and the incidence of dementia. Thus, reporting a medium number of VE symptoms was associated with a $25 \%$ higher incidence of dementia $(\mathrm{IRR}=1.250 ; 95 \% \mathrm{CI}: 1.015-1.540)$, while reporting a high number of VE symptoms was associated with a $40 \%$ higher incidence of dementia (IRR $=1.404 ; 95 \%$ CI: 1.049-1.878). However, the association between reporting a low number of VE symptoms and the incidence of dementia was not statistically significant (IRR $=1.164$; 95\% CI: 0.994-1.363). Adjusting for lifestyle factors and comorbidities did not attenuate the association between levels of VE symptoms and the risk of dementia.

In Table 3, the results from the analyses with the continuous VE variable showed that for every one-symptom increase in VE, the IRR of dementia increased by $2 \%$ (IRR $=1.023$; 95\% CI: $1.004-1.043$ ) after adjusting for socio-demographic factors and mental disorders (Model 2). When further adjusting for lifestyle factors and comorbidities, the dementia
IRR increased with $3 \%$ for every one-symptom increase in VE. Thus, adjustment did not attenuate the association between VE and dementia.

The results of the stratified analyses did not reveal substantial differences in the estimates of the associations between VE and dementia among those aged $<60$ years versus $\geq 60$ years or among those being unmarried versus married (Table 4 ). The results were similar when using $<70$ years and $\geq 70$ years as a cut-off (data not shown). The estimates of the association between VE and dementia seemed to be somewhat stronger among men than among women, and whereas the estimates for the groups with low and medium education supported our overall findings, the estimates for the group with high education did not support that VE was associated with a higher incidence of dementia. Due to the lower statistical power in the stratified analyses, only the estimates for men and for the group with medium educational level reached statistical significance at conventional levels.

\section{Sensitivity analyses}

When postponing the start of follow-up to 10, 15, or 20 years after the VE assessment, identical IRR's 

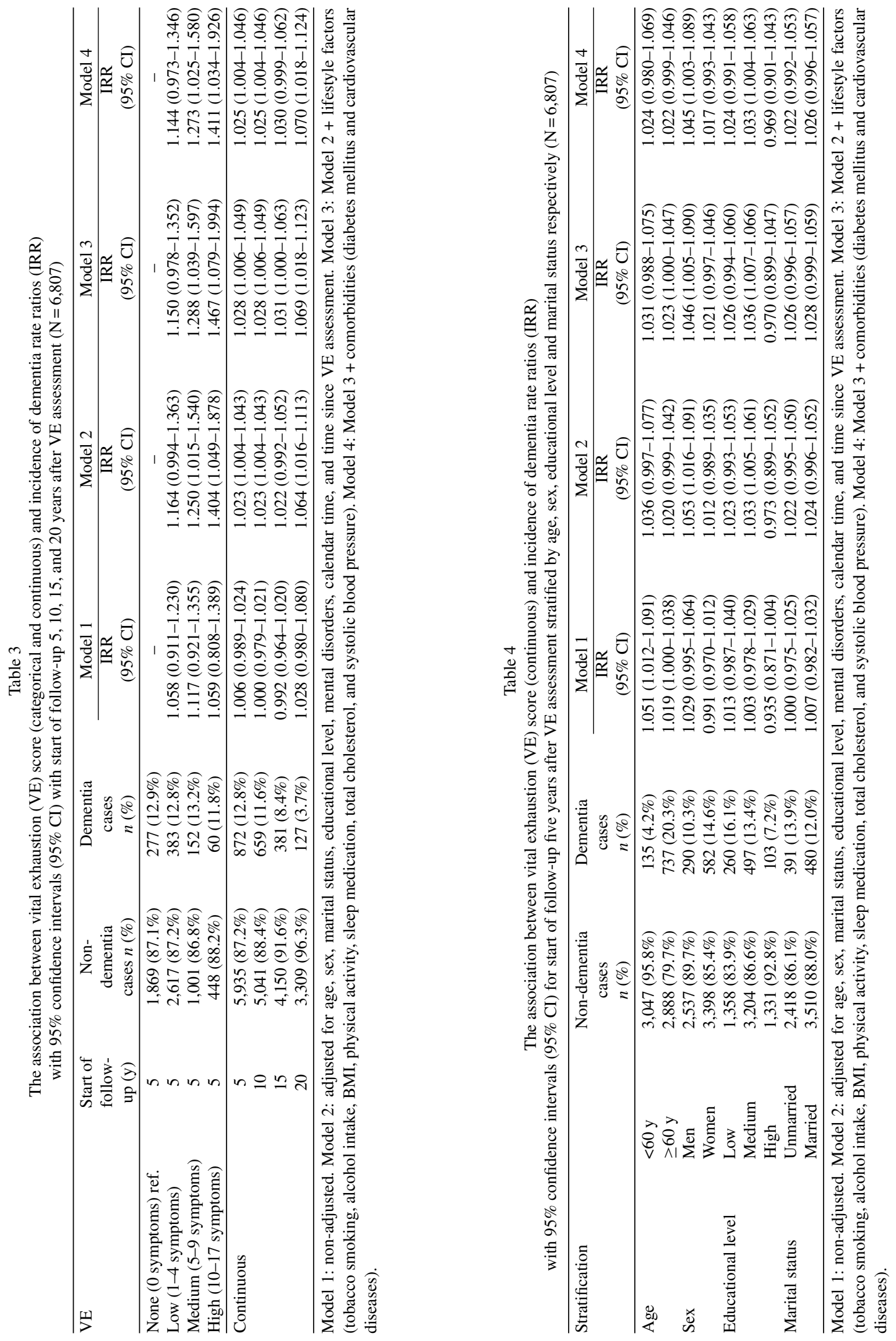
of dementia were observed as when starting followup of dementia from five years after VE assessment (Table 3). Thus, the sensitivity analyses did not alter the previous results on the associations between VE and incidence of dementia.

Our additional analyses investigating the association between reporting VE at baseline in 1991-1994 and again in the next survey in 2001-2003 showed a Pearson correlation coefficient of $r=0.523, p<0.001$ $(n=3,784)$. Thus, the retest correlation was quite substantial with a 10-year retest interval. By using frequency analyses and analyzing VE in the four categories displayed in Tables 2 and 3, we investigated whether participants who participated in both surveys changed their level of VE from the first survey to the next. We observed that $30 \%$ reported a higher VE level than previously, 20\% reported a lower VE level than previously, and 50\% reported the same level of VE at both time points (data not shown).

\section{DISCUSSION}

Among the 6,807 participants of this longitudinal, population-based study, we found a positive association between the number of VE symptoms and the incidence of dementia. Even when postponing the start of follow-up to 10,15, and 20 years after VE was reported, the direction and the magnitude of the association between VE and the incidences of dementia remained consistent.

Adjustment for lifestyle factors and comorbidities did not change the results substantially in any of the analyses. Stratifying the analyses by age, sex, educational level and marital status did not reveal different associations between VE and dementia when compared with the association observed for the whole population. One exception was the group with high educational level for whom we found no indication of a higher incidence of dementia with increasing VE. There was a positive and significant correlation between how participants reported VE at baseline and again 10 years later, and we cannot rule out that VE at least partly may reflect a personality trait rather than a mental state.

Our findings are strengthened by the large study population, the use of a validated VE scale, and the low risk of loss to follow-up as all participants were followed in national registers and only 3 participants were lost to follow-up due to emigration. Furthermore, the large amount of both self-reported and register data made it possible to adjust for several important risk factors for dementia. By excluding participants with a dementia diagnosis during the first five years after the assessment of VE, we aimed at reducing reverse causation. Even when we further postponed start of follow-up, the observed association remained. However, it is important to consider that the association between VE and dementia might have been diluted due to the long time-span between exposure and outcome assessment. This could be the case particularly for participants who did not report the same number of VE symptoms 10 years later, which was the case for $50 \%$ of the participants.

VE represents subjective accounts of the participants' feelings in the past six months. Hence, their general way of perceiving themselves and their surroundings will most likely affect how they respond. Psychological distress could be due to higher vulnerability to stressors and poor coping strategies $[4$, $5,38]$. Experiencing several stressors that potentially accumulate over time can cause psychological distress in individuals depending on how they perceive stressors $[39,40]$. Being high on the personality trait neuroticism and being prone to experiencing psychological distress have been shown to be associated with the risk of dementia [41]. Among healthy individuals reporting VE, a longitudinal association was found between VE and neuroticism, low self-esteem, and a tendency to use an emotion-oriented coping strategy in stressful situations [42]. Based on this knowledge, it cannot be ruled out that the measure of VE reflects a stable personality trait rather than psychological distress. Alternatively, being high on personality traits, such as neuroticism, could make some individuals more prone to developing VE as a response to external stressors.

A possible, unmeasured confounder is the apolipoprotein E (ApoE) genotype, which is involved in sporadic dementia and which has been observed to affect the modulation of stress response processes [43]. We cannot rule out that reporting high VE scores is associated with the ApoE gene which would create a spurious association between VE and dementia. Other potential confounders could be other established risk factors for dementia, such as hearing loss and social isolation [2].

Despite the high validity of dementia diagnoses and the improvement of the diagnostic rate in Denmark [44, 45], approximately $60 \%$ of dementia cases are not diagnosed, which could lead to biased results $[46,47]$. Suggested reasons for not being diagnosed could be the patient's fear of stigmatization, lack of knowledge of dementia and treatment among patients 
and relatives, clinicians lacking clinical diagnostic guidelines, and varying diagnostic procedures at different hospitals $[44,46]$. Thus, register data on dementia diagnoses underestimate the prevalence in the general population. To improve the validity of our outcome measure, we also added information about prescription of anti-dementia medication. Yet, this choice meant that some patients with dementia in Parkinson's disease might erroneously be categorized as cases in the present study.

In CCHS as a whole, $61.2 \%$ of those invited responded to the third survey. In the age group relevant for this study, the response rate ranged from $52.1-67.6 \%$ among women in which the lowest rate was in the age group 75-79 years and highest in the age group 50-54 years. For men, the response rate range was $56.5-64.0 \%$, and the rate was lowest in the age group 35-39 years and highest in the age group 50-54 years [48]. Participants were included depending on their morbidity (i.e., being non-demented) and mortality (i.e., surviving until the survey in 1991-1994 and start of follow-up). As a consequence, included participants are most likely healthier than non-participants. Altogether, these analytical decisions and the selection of healthy individuals into the survey are likely to yield an underestimation of the true association between VE and dementia. Yet, it is also possible that individuals with poor health are more likely to be in contact with the healthcare system and be registered with a dementia diagnosis. Indeed, our descriptive analyses showed that those reporting high VE seemed to be disadvantaged in terms of their health status, which then may imply a potential overestimation of the association between VE and dementia.

This is the first study investigating the association between VE and incidence of dementia. However, our results are in line with previous studies of psychological distress and dementia [4-10]. Furthermore, the similarity between symptoms of depression and VE also supports the potential link between VE and dementia, as depression is a well-established risk factor for dementia [2, 25, 49]. At the same time, the similarity between symptoms of depression and VE adds to the complexity of distinguishing between the concepts, their symptoms and their origin. Therefore, we cannot eliminate the possibility that symptoms of VE could be symptoms of underlying depression. Thus, residual confounding due to the presence of depression at the time of VE assessment is still likely to influence the results. Another study found that especially the depressive feelings of hopelessness were associated to cognitive health and the risk of $\mathrm{AD}$ and that immunology or lifestyle factors could be potential mechanisms [50]. Several of the VE symptoms relate to both the hopelessness dimension of depression, but also the somatic dimension, thus, there is an overlap of symptoms between the two concepts $[20,50]$.

Conceptually, symptoms of VE also overlaps with burnout, which is a condition caused by long-term exposure to occupational stressors $[19,51]$. VE is not conceptualized as a response to specific stressors and the different tools of assessing VE and burnout make it difficult to compare burnout to VE [19]. However, since we have no information on what type of stressors led to VE, we cannot rule out that VE could reflect burnout in some of the participants.

Subclinical symptoms of depression have shown to impact cognitive function negatively [52]. However, it has also been suggested that depressive symptoms and depression diagnoses in later life might be early manifestations of dementia [2]. Since symptoms of VE and depression have shown to overlap [25], VE could be a prodromal sign of dementia. In combination with the long preclinical phase of $\mathrm{AD}$, in particular, there is a risk of reverse causation. Importantly, the association between VE and dementia remained when postponing the start of follow-up, which supports the notion that our findings are not solely explained by VE being an early sign of pathological changes eventually leading to dementia.

We acknowledge previous critical perspectives on $\mathrm{VE}$ as being a tool for research rather than practice and prevention, and that this is an older concept used in previous questionnaires [53]. However, data on VE have also been collected in recent studies and is still used in today's research $[19,20]$. Research on midlife risk factors and health outcomes in later life depends on long follow-up periods in which use of previously applied and maybe debated concepts are sometimes the only option in today's research.

\section{Conclusions}

In this study, we found arguments that VE is a risk factor for dementia. After adjusting for important confounders, the associations became significant and remained unaffected when addressing potential reverse causation. However, as VE seemed to be relatively stable over a 10-year period in half of the participants, VE could reflect personality types of individuals who are also more prone to experiencing negative health outcomes in older age. Our 
results highlight the importance of identifying and considering indicators of psychological distress in the prevention of later disease. As this is the first study on VE and dementia, more longitudinal and interdisciplinary research is needed to understand the underlying mechanism between psychological distress in general, VE more specifically, and neurological diseases such as dementia.

\section{ACKNOWLEDGMENTS}

We thank Ellen Garde, Medical Doctor and Associate Professor from Center for Healthy Aging at the University of Copenhagen, for insightful discussions, which greatly assisted the research.

Financial support for this research was funded as part of the corresponding author's $\mathrm{PhD}$ project by VELUXFONDEN (00014847).

Authors' disclosures available online (https:// www.j-alz.com/manuscript-disclosures/18-0478r2).

\section{REFERENCES}

[1] American Psychiatric Association (2013) Diagnostic and Statistical Manual of Mental Disorders (DSM-5), American Psychiatric Publishing, Washington, DC.

[2] Livingston G, Sommerlad A, Orgeta V, Costafreda S, Huntley J, Ames D, Ballard C, Banerjee S, Burns A, CohenMansfield J, Cooper C, Fox N, Gitlin L, Howard R, Kales H, Larson E, Ritchie K, Rockwood K, Sampson E, Samus Q, Schneider L, Selbæk G, Teri L, Mukadam N (2017) Dementia prevention, intervention, and care. Lancet 390, 2673-2734.

[3] Prince M, Wimo A, Guerchet M, Ali G, Wu Y, Prina M (2015) World Alzheimer Report 2015: The Global Impact of Dementia - An analysis of prevalence, incidence, cost and trends. Alzheimer's Disease International. https:// www.alz.co.uk/research/WorldAlzheimerReport2015.pdf

[4] Johansson L, Guo X, Waern M, Östling S, Gustafson D, Bengtsson C, Skoog I (2010) Midlife psychological stress and risk of dementia: A 35-year longitudinal population study. Brain 133, 2217-2224.

[5] Skogen J, Bergh S, Stewart R, Knudsen A, Bjerkeset O (2015) Midlife mental distress and risk for dementia up to 27 years later: The Nord-Trøndelag Health Study (HUNT) in linkage with a dementia registry in Norway. BMC Geriatr $15,23$.

[6] Greenberg M, Tanev K, Marin M, Pitman R (2014) Stress, PTSD, and dementia. Alzheimers Dement 10, S155-S165.

[7] Crowe M, Andel R, Pedersen N, Gatz M (2007) Do workrelated stress and reactivity to stress predict dementia more than 30 years later? Alzheimer Dis Assoc Disord 21, 205209.

[8] Wilson R, Arnold S, Schneider J, Kelly J, Tang Y, Bennett D (2006) Chronic psychological distress and risk of Alzheimer's disease in old age. Neuroepidemiology 27, 143153.
[9] Wilson R, Evans D, Bienias J, Mendes de Leon C, Schneider J, Bennett D (2003) Proneness to psychological distress is associated with risk of Alzheimer's disease. Neurology 61, 1479-1485.

[10] Simard M, Hudon C, van Reekum R (2009) Psychological distress and risk for dementia. Curr Psychiatry Rep 11, 41-47.

[11] Drapeau A, Marchand A, Beaulieu-Prévost D (2012) Epidemiology of psychological distress. In Mental Illnesses - Understanding, Prediction and Control, LAbate L, ed. IntechOpen, pp. 105-134.

[12] Sandi C (2007) Memory impairments associated with stress and aging. In Neural Plasticity and Memory: From Genes to Brain Imaging, Bermúdez-Rattoni F, ed. CRC Press/Taylor \& Francis, Boca Raton, FL, USA.

[13] Tolppanen A, Solomon A, Soininen H, Kivipelto M (2012) Midlife vascular risk factors and Alzheimer's disease: Evidence from epidemiological studies. J Alzheimers Dis 32, 531-540.

[14] Seeman TS, BH, Rowe J, Horwitz R, McEwen B (1997) Price of adaptation-allostatic load and its health consequences: MacArthur Studies of Successful Aging. Arch Intern Med 157, 2259-2268.

[15] Seeman T, McEwen B, Singer B, Albert M, Rowe J (1997) Increase in urinary cortisol excretion and memory declines: MacArthur studies of successful aging. J Clin Endocrinol Metab 82, 2458-2465.

[16] Appels A, Höppener P, Mulder P (1987) A questionnaire to assess premonitory symptoms of myocardial infarction. Int J Cardiol 17, 15-24.

[17] Appels A, Falger PR, Schouten EG (1993) Vital exhaustion as risk indicator for myocardial infarction in women. J Psychosom Res 37, 881-890.

[18] van Diest R, Appels A (2002) Vital exhaustion: Behavioural and biological correlates. Curr Opin Psychiatry 15, 639641.

[19] Frestad D, Prescott E (2017) Vital exhaustion and coronary heart disease risk: A systematic review and meta-analysis. Psychosom Med 79, 260-272.

[20] Balog P, Falger P, Szabó G, Rafael B, Székely A, Konkolÿ Thege B (2017) Are vital exhaustion and depression independent risk factors for cardiovascular disease morbidity? Health Psychol 36, 740-748.

[21] Pedersen J, Lund R, Andersen I, Clark A, Prescott E, Rod N (2016) Psychosocial risk factors for the metabolic syndrome: A prospective cohort study. Int J Cardiol $\mathbf{2 1 5}$, 41-46.

[22] Krummenacher R, Lukas P, Biasiutti F, Begré S, Znoj H, Von Känel R (2009) Independent association of sleep quality, fatigue, and vital exhaustion with platelet count in patients with a previous venous thromboembolic event. Platelets 20, 566-574.

[23] Clark A, Strandberg-Larsen K, Masters Pedersen J, Lange P, Prescott E, Rod N (2015) Psychosocial risk factors for hospitalisation and death from chronic obstructive pulmonary disease: A prospective cohort study. COPD 12, 190-198.

[24] Iversen L, Strandberg-Larsen K, Prescott E, Schnohr P, Rod N (2012) Psychosocial risk factors, weight changes and risk of obesity: The Copenhagen City Heart Study. Eur J Epidemiol 27, 119-130.

[25] Kop W (2012) Somatic depressive symptoms, vital exhaustion, and fatigue: Divergent validity of overlapping constructs. Psychosom Med 74, 442-445. 
[26] van Diest R, Appels A (1991) Vital exhaustion and depression: A conceptual study. J Psychosom Res 35, 535-544.

[27] Johansson L (2014) Can stress increase Alzheimer's disease risk in women? Expert Rev Neurother 14, 123-125.

[28] Aguib Y, Al Suwaidi J (2015) The Copenhagen City Heart Study (Østerbroundersøgelsen). Glob Cardiol Sci Pract 2015, 1-15.

[29] Salem L, Andersen B, Nielsen T, Stokholm J, Jorgensen M, Rasmussen M, Waldemar G (2012) Overdiagnosis of dementia in young patients - a nationwide register-based study. Dement Geriatr Cogn Disord 34, 292-299.

[30] Nielsen T, Vogel A, Phung T, Gade A, Waldemar G (2011) Over- and under-diagnosis of dementia in ethnic minorities: A nationwide register-based study. Int J Geriatr Psychiatry 26, 1128-1135.

[31] Prescott E, Holst C, Gronbaek M, Schnohr P, Jensen G, Barefoot J (2003) Vital exhaustion as a risk factor for ischaemic heart disease and all-cause mortality in a community sample. A prospective study of 4084 men and 5479 women in the Copenhagen City Heart Study. Int J Epidemiol 32, 990-997.

[32] Lynge E, Sandegaard J, Rebolj M (2011) The Danish National Patient Register. Scand J Public Health 39, 30-33.

[33] Mors O, Perto G, Mortensen P (2011) The Danish Psychiatric Central Research Register. Scand J Public Health 39, 54-57.

[34] Helweg-Larsen K (2011) The Danish Register of Causes of Death. Scand J Public Health 39, 26-29.

[35] Kildemoes H, Sørensen H, Hallas J (2011) The Danish National Prescription Registry. Scand J Public Health 39, $38-41$.

[36] Sommerlad A, Ruegger J, Singh-Manoux A, Lewis G, Livingston G (2018) Marriage and risk of dementia: Systematic review and meta-analysis of observational studies. J Neurol Neurosurg Psychiatry 89, 231-238.

[37] Pase M, Himali J, Grima N, Beiser A, Satizabal C, Aparicio H, Thomas R, Gottlieb D, Auerbach S, Seshadri S (2017) Sleep architecture and the risk of incident dementia in the community. Neurology 89, 1244-1250.

[38] Johansson L, Guo X, Hällström T, Norton M, Waern M, Östling S, Bengtsson C, Skoog I (2013) Common psychosocial stressors in middle-aged women related to longstanding distress and increased risk of Alzheimer's disease: A 38-year longitudinal population study. BMJ Open 3, e003142.

[39] Miller T (1993) The assessment of stressful life events. In Handbook of stress, theoretical and clinical aspects, Goldberger L, Breznitz S, eds. Free Press, New York, US, pp. 161-173.
[40] Rod N, Andersen I, Prescott E (2011) Psychosocial risk factors and heart failure hospitalization: A prospective cohort study. Am J Epidemiol 174, 672-680.

[41] Low L, Harrison F, Lackersteen S (2013) Does personality affect risk for dementia? A systematic review and metaanalysis. Am J Geriatr Psychiatry 21, 713-728.

[42] van Zijderveld G, Twisk J, Boers M, Cuijpers P (2013) Relation between personality, coping style and vital exhaustion Neth J Psychol 68, 35-42.

[43] Dose J, Huebbe P, Nebel A, Rimbach G (2016) APOE genotype and stress response - a mini review. Lipids Health Dis 15, 1-15.

[44] Phung T, Andersen B, Hogh P, Kessing L, Mortensen P, Waldemar G (2007) Validity of dementia diagnoses in the Danish Hospital Registers. Dement Geriatr Cogn Disord 24, 220-228.

[45] Phung TKT, Waltoft BL, Kessing LV, Mortensen PB, Waldemar G (2010) Time trend in diagnosing dementia in secondary care. Dement Geriatr Cogn 29, 146-153.

[46] Jørgensen K, Waldemar G (2015) Prævalens af demens i Danmark. Ugeskr Laeger 176, 1041-1044.

[47] Prince M, Bryce R, Ferri C (2011) World Alzheimer Report 2011: The benefits of early diagnosis and intervention. Alzheimer's Disease International.

[48] Schnohr P, Jensen G, Lange P, Scharling H, Appleyard M (2001) The Copenhagen City heart study - Introduction. Eur Heart J Suppl 3, H1-H83.

[49] McGowan L, Dickens C, Percival C, Douglas J, Tomenson B, Creed F (2004) The relationship between vital exhaustion, depression and comorbid illnesses in patients following first myocardial infarction. J Psychosom Res 57, 183-188.

[50] Håkansson K, Soininen H, Winblad B, Kivipelto M (2015) Feelings of hopelessness in midlife and cognitive health in later life: A prospective population-based cohort study. PLoS One 10, e0140261.

[51] Toker S, Melamed S, Berliner S, Zeltser D, Shapira I (2012) Burnout and risk of coronary heart disease: A prospective study of 8838 employees. Psychosom Med 74, 840-847.

[52] Brevik E, Eikeland R, Lundervold A (2013) Subthreshold depressive symptoms have a negative impact on cognitive functioning in middle-aged and older males. Front Psychol 4, 309.

[53] Luepker R, Schulz S (2015) Vital exhaustion: Ready for prime time? Eur Heart J 36, 1361-1363. 